\title{
HOMOTOPY FOR FUNCTORS
}

\author{
MING-JUNG LEE
}

\begin{abstract}
We show that natural transformations play the role of homotopy for (covariant) functors. Homotopic functors are shown to induce identical maps between the homology groups of categories. For a space $X$, there is an associated category $\Lambda S(X)$. We show that the classifying space of $\Lambda S(X)$ has the same homotopy type as $X$ if $X$ is a $\mathrm{CW}$ complex. Moreover, we prove that, for $\mathrm{CW}$ complexes $X$ and $Y, f$ and $g: X \rightarrow Y$ are homotopic if and only if $\Lambda S(f)$ and $\Lambda S(g)$ are.
\end{abstract}

1. Introduction. It is known that a simplicial complex is topologically determined by a partially ordered set. In particular, this partially ordered set determines the homotopy type of the simplicial complex. It is also known that the homotopy type of a $\mathrm{CW} K(G, n)$ is determined by the group $G$. Partially ordered sets and groups are related in the sense that they both are small categories. In this paper, we show that the homotopy type of a $\mathrm{CW}$ complex is determined by a small category, the singular category of the complex, where natural transformations play the role of homotopy for (covariant) functors. Our approach is closely related to simplicial sets. Functors will always be covariant.

Let $K$ be a simplicial set. We associate to $K$ a small category $\Lambda(K)$ as follows. The objects of $\Lambda(K)$ are simplexes of $K$. The set of morphisms from simplex $v$ to simplex $u$ consists of all the face maps $x$ from $\Delta^{m}$ to $\Delta^{n}$, where $m=\operatorname{dim} v$ and $n=\operatorname{dim} u$, such that $u x=v$. (A face map is an injective monotone function from $[m]=\{0,1, \cdots, m\}$ to $[n]=\{0,1, \cdots, n\}$ and it operates on $u$ via the face maps of $K$.) For a simplicial map $f: K \rightarrow L$, we define $\Lambda(f)=\Lambda(K) \rightarrow \Lambda(L)$ to be the obvious functor with $\Lambda(f)(u)=$ $f(u)$ for objects. Thus $\Lambda$ becomes a functor from $\mathscr{S}$, the category of simplicial sets, to $\mathscr{C}$, the category of small categories and functors. Composing $\Lambda$ with $S$, the total singular complex functor, one obtains a functor from $\mathscr{T}$, the category of topological spaces, to $\mathscr{S}$. The category $\Lambda S(X)$ is called the singular category of the space $X$. We prove the functor $\Lambda S$ preserves homotopy.

Presented to the Society, January 19, 1972; received by the editors March 1, 1972. AMS (MOS) subject classifications (1970). Primary 55D10, 55D15; Secondary 18A99. Key words and phrases. Small category, simplicial set, homotopy, subdivision, morphism complex, classifying space.

(c) American Mathematical Society 1973 
There is also a functor $M$ from $\mathscr{C}$ to $\mathscr{S}$ which preserves homotopy. The functor $M$ was studied by Anderson [1] and Segal [7]. In general $M(\Lambda)$ may not be a Kan complex (where $\Lambda$ is any category); we prove that $M(\Lambda)$ is a Kan complex if and only if $\Lambda$ is a groupoid. We also show $M \Lambda(K)$ is precisely the subdivision (see Kan [2]) of the simplicial set $K$. For a category $\Lambda$, the geometric realization of $M(\Lambda)$ is called the classifying space of $\Lambda$ and denoted by $B \Lambda$. It is well known that the classifying space of a group $G$ is a CW $K(G, 1)$ and the classifying spaces of finite partially ordered sets are simplicial complexes. We prove that the classifying space of the category $\Lambda S(X)$ has the same homotopy type as $X$ if $X$ is a $\mathrm{CW}$ complex.

Our main theorem can be stated as follows.

THEOREM 1. Let $X$ and $Y$ be two $\mathrm{CW}$ complexes.

(a) For any two maps $f$ and $g: X \rightarrow Y, f \simeq g$ if and only if $\Lambda S(f) \simeq \Lambda S(f)$.

(b) $X$ and $Y$ have the same homotopy type if and only if $\Lambda S(X)$ and $\Lambda S(Y)$ have the same homotopy type.

We can also show that homotopic functors induce same maps between the homology groups of small categories with constant coefficients. (It was shown in Lee [3] that the homology groups of a space $X$ coincide with the homology groups of its singular category.)

The author would like to express his appreciation to Professor Charles Watts for his help and encouragement.

2. Relations between small categories and simplicial sets.

Definition 2. Let $\Lambda$ be a small category. We define the morphism complex $M \Lambda$ of $\Lambda$ to be a simplicial set where

$$
\begin{aligned}
& M_{0} \Lambda=\{(u) \mid u \text { is an object of } \Lambda\}, \\
& M_{p} \Lambda=\left\{\left[x_{p-1}, \cdots, x_{0}\right] \mid x_{i} \text { is a morphism in } \Lambda\right. \text { and } \\
&\left.x_{p-1} \circ \cdots \circ x_{0} \text { is defined }\right\}, \quad p \geqq 1 .
\end{aligned}
$$

(We use $u$ for both object and identity morphism, thus $(u) \in M_{0} \Lambda$ and $[u] \in M_{1} \Lambda$.) Face and degeneracy operators are defined by

and

$$
\partial_{i}\left[x_{p-1}, \cdots, x_{0}\right]=\left[x_{p-1}, \cdots, x_{i} \circ x_{i-1}, \cdots, x_{0}\right]
$$

$$
s_{i}\left[x_{p-1}, \cdots, x_{0}\right]=\left[x_{p-1}, \cdots, x_{i}, u_{i}, x_{i-1}, \cdots, x_{0}\right]
$$

for $p>1$ where $u_{i}=$ Domain $x_{i}=$ Range $x_{i-1}$. For $p=1$, we let $\partial_{0}\left[x_{0}\right]=\left(u_{0}\right)$, $\partial_{1}\left[x_{0}\right]=\left(u_{1}\right)$ and $s_{0}(u)=[u]$.

It is not hard to check that $M \Lambda$ is indeed a simplicial set. For a functor $f: \Lambda \rightarrow \Gamma$ of small categories, we let $M f: M \Lambda \rightarrow M \Gamma$ be the simplicial map defined by $M_{p}(f)\left[x_{p-1}, \cdots, x_{0}\right]=\left[f\left(x_{p-1}\right), \cdots, f\left(x_{0}\right)\right]$. It is readily verified that $M$ is in fact a functor from $\mathscr{C}$ to $\mathscr{S}$. 
In general, $M \Lambda$ may not be a Kan complex (e.g. when $\Lambda$ is a partially ordered set). However, $M \Lambda$ is a Kan complex if $\Lambda$ is a groupoid. In fact, we have

THEOREM 3. M $\Lambda$ is a Kan complex (i.e., satisfies the extension condition) if and only if $\Lambda$ is a groupoid.

Proof. (a) Only if part: We will show that if $z$ and $y$ are morphisms of $\Lambda$ with Range $z=$ Range $y$ or Domain $z=$ Domain $y$ then there is a morphism $x$ such that $z x=y$ or $x z=y$ respectively. Suppose Range $z=$ $u=$ Range $y$. Consider $\sigma_{0}=[z]$ and $\sigma_{1}=[y]$ in $M_{1} \Lambda$. Since $\partial_{0} \sigma_{1}=\partial_{0} \sigma_{0}$, by the extension condition there is a $\left[x_{1}, x_{0}\right]$ in $M_{2} \Lambda$ such that $\left[x_{1}\right]=$ $\partial_{0}\left[x_{1}, x_{0}\right]=\sigma_{0}=[z]$ and $\left[x_{1} x_{0}\right]=\partial_{1}\left[x_{1}, x_{0}\right]=\sigma_{1}=[y]$. Hence $x_{1}=z$ and $x_{1} x_{0}=y$, i.e., there is an $x=x_{0}$ with $z x=y$. The other part can be similarly proved by considering $\sigma_{1}=[y]$ and $\sigma_{2}=[z]$.

(b) If part: Let $\sigma_{0}, \cdots, \sigma_{k-1}, \sigma_{k\lrcorner 1}, \cdots, \sigma_{p+1}$ be simplexes of $M_{p} \Lambda$ $\left(p \geqq 1\right.$ ) which satisfy the compatibility conditions $\partial_{i} \sigma_{j}=\partial_{j-1} \sigma_{i}$ for $i<j$, $i \neq k$ and $j \neq k$. Write

$$
\sigma_{j}=\left[x_{n-1}^{j}, \cdots, x_{0}^{j}\right] .
$$

We may suppose $k \neq 0$ (if $k=0$, then $k \neq p+1$ and the proof proceeds in a similar way). Since $\partial_{0} \sigma_{j}=\partial_{j-1} \sigma_{0}$ for $j>0$ and $j \neq k$, we have

$$
\begin{aligned}
\sigma_{j} & =\left[x_{p-1}^{0}, \cdots, x_{j-1}^{0}, x_{j-2}^{0}, \cdots, x_{0}^{0}, x_{0}^{j}\right] \text { for } 2 \leqq j \leqq p \text { and } j \neq k \\
\sigma_{1} & =\left[x_{p-1}^{0}, \cdots, x_{1}^{0}, x_{0}^{1}\right] \text { if } k \neq 1 \text { and } \\
\sigma_{p-1} & =\left[x_{p-2}^{0}, \cdots, x_{0}^{0}, x_{0}^{p+1}\right] \text { if } k \neq p+1 .
\end{aligned}
$$

We divide our proof into three parts.

(1) Suppose $p \geqq 3$. Then there is a number $l \geqq 3$ and $k \neq l$ and it follows from the compatibility conditions $\left(\partial_{l} \sigma_{j}=\partial_{j-1} \sigma_{l}\right.$ for $l<j$ and $\partial_{j} \sigma_{l}=\partial_{l-1} \sigma_{j}$ for $j<l)$ that

and

$$
x_{0}^{j}=x_{0}^{l} \text { for } 2 \leqq j \leqq p+1, \quad j \neq k,
$$

$$
x_{0}^{1}=x_{0}^{0} x_{0}^{l} \quad \text { if } k \neq 1 \quad\left(\text { use } \partial_{1} \sigma_{l}=\partial_{l-1} \sigma_{1} \text { and } \partial_{0} \sigma_{l}=\sigma_{l-1} \sigma_{0}\right) .
$$

Denote $x_{0}^{l}$ by $x_{0}, x_{j-1}^{0}$ by $x_{j}$. Then the $(p+1)$-simplex $\sigma=\left[x_{p}, \cdots, x_{0}\right]$ has the properties that $\partial_{j} \sigma=\sigma_{j}$ for all $j \neq k$.

(2) Suppose $p=2$. Then there is a number $l \geqq 2$ and $k \neq l$. If $l=3$, then the proof is the same as case (1). If $l=2$, then again by the compatibility conditions we have

and

$$
x_{0}^{j}=x_{0}^{2} \text { for } 2 \leqq j \leqq p+1, \quad j \neq k,
$$

$$
x_{1}^{0} x_{0}^{1}=x_{1}^{0} x_{0}^{0} x_{0}^{2} \quad \text { if } k \neq 1 \quad \text { (use also } \partial_{0} \sigma_{1}=\partial_{0} \sigma_{0} \text { ) }
$$


Since $\Lambda$ is groupoid, $x_{1}^{0} x_{0}^{1}=x_{1}^{0} x_{0}^{0} x_{0}^{2}$ implies $x_{0}^{1}=x_{0}^{0} x_{0}^{2}$ and the proof coincides with that of (1).

(3) Suppose $p=1$. Then the proof is the reverse of the arguments in part (a).

(In fact, we have shown that the extension condition is always satisfied for $p \geqq 3$.) Q.E.D.

We now show that the classifying space of $\Lambda S(X)$ has the same homotopy type as $X$ if $X$ is a CW complex. We first observe that for any simplicial set $K$ the simplicial set $S \Lambda(K)$ is just the subdivision $S d K$ (see Kan [2]). In fact, $S d K$ is defined to be the simplicial set $\bar{K} / \sim$, where $\bar{K}_{p}=$ $\left\{\left[u, x_{p}, \cdots, x_{0}\right] \mid u \circ x_{p} \circ \cdots \circ x_{0}\right.$ defined in $\left.\Lambda(K)\right\}$ and $\left[u, x_{p}, \cdots, x_{0}\right] \sim$ $\left[v, y_{p}, \cdots, y_{0}\right]$ if $y_{i}=x_{i}$ for $i=0,1, \cdots, p-1$ and $u x_{p}=v y_{p}$. Therefore, the simplicial map $q: S d K \rightarrow S \Lambda(K)$ with $\varphi_{p}\left[u, x_{p}, \cdots, x_{0}\right]=\left[x_{n-1}, \cdots, x_{0}\right]$ is an isomorphism and

Proposition 4. $S d K$ and $S \Lambda(K)$ are isomorphic as simplicial sets.

PROPOSITION 5. There is a natural transformation $D$ from the functor $B \Lambda S=T M \Lambda S: \mathscr{T} \rightarrow \mathscr{T}$ to the functor $1_{\mathscr{T}}$ such that $D(X): B \Lambda S(X) \rightarrow X$ is a weak homotopy equivalence for every topological space $X$ and hence $a$ homotopy equivalence if $X$ is a $\mathrm{CW}$ complex. ( $T$ is the geometric realization functor.)

Proof. By Kan [2], there is a natural transformation $d$ from $S d$ to $1_{s}$. Composing $d$ with $T$ and $S$, we obtain a natural transformation $d^{\prime}$ from $T S d S$ to $T S$. On the other hand, there is an adjunction natural transformation $\psi$ from $T S$ to $1_{\mathscr{T}}$ (cf. May [5]). Combining with the isomorphism of Proposition 4, we get a natural transformation $D=\psi \circ d^{\prime}: B \Lambda S=$ $T M \Lambda S \rightarrow 1_{\mathscr{T}}$. The map $D(X): B \Lambda S(X) \rightarrow X$ is a weak homotopy equivalence because both $d^{\prime}$ and $\psi$ are weak homotopy equivalences (see Kan [2] and May [5]). Q.E.D.

\section{Invariance of homotopies.}

Definition 6. Let $\varphi$ and $\varphi^{\prime}: \Lambda \rightarrow \Gamma$ be covariant functors of small categories. We say that $\varphi$ is homotopic to $\varphi^{\prime}$ if there are covariant functors $\varphi_{i}=\Lambda \rightarrow \Gamma, i=0,1, \cdots, n$, with $\varphi_{0}=\varphi$ and $\varphi_{n}=\varphi^{\prime}$ such that for each $i$ there is a natural transformation between $\varphi_{i}$ and $\varphi_{i+1}$. Homotopy will be denoted by $\simeq$.

REMARK. The homotopy relation defined above is obviously an equivalence relation. It is also easy to see that $\varphi \simeq \varphi^{\prime}$ and $\psi \simeq \psi^{\prime}$ imply $\varphi \circ \psi \simeq \varphi^{\prime} \circ \psi^{\prime}$. In case $\Lambda$ and $\Gamma$ are partially ordered sets, the homotopy coincides with the one defined by Okamoto [6]. For monoids $\Lambda$ and $\Gamma$, two functors (i.e., homomorphisms) $\varphi$ and $\varphi^{\prime}$ are homotopic if there are 
$y_{i}$ and $\varphi_{i}$ for $i=0,1, \cdots, n$ such that $q=\varphi_{0}, \varphi^{\prime}=\varphi_{n}$ and $y_{i} \circ \varphi_{i}(x)=$ $\varphi_{i+1}(x) \circ y_{i}$. Furthermore, if $\Gamma$ is a group, then $\varphi$ and $\phi^{\prime}$ are homotopic if and only if there is a $y \in \Gamma$ with $\varphi(x)=y^{-1} \varphi(x) y$, i.e., homotopy coincides with conjugation.

Proposition 7. If $\varphi$ and $\varphi^{\prime}: \Lambda \rightarrow \Gamma$ are homotopic functors, then $M \varphi$ and $M \varphi^{\prime}$ are also homotopic as simplicial maps.

Proof. This proposition was proved by G. Segal [7] for topological categories. We will give a different proof here. Suppose $\eta$ is a natural transformation from $\varphi^{\prime}$ to $\varphi$, we construct a homotopy $h=M \varphi^{\prime} \simeq M \varphi$ as follows. For each $p \geqq 0$ and $0 \leqq j \leqq p$, we define $h_{j}: M_{p} \Lambda \rightarrow M_{p+1} \Gamma$ by

$$
h_{j}\left[x_{p-1}, \cdots, x_{0}\right]=\left[\varphi\left(x_{p-1}\right), \cdots, \varphi\left(x_{j}\right), \eta\left(u_{j}\right), \varphi^{\prime}\left(x_{j-1}\right), \cdots, \varphi^{\prime}\left(x_{0}\right)\right]
$$

where $u_{j}=$ Domain $x_{j}=$ Range $x_{j-1}$ (for $p=0, h_{0}(u)=[\eta(u)]$ ). Using the hypothesis that $\eta$ is a natural transformation, one can check $h$ is indeed a homotopy between $\varphi^{\prime}$ and $\varphi$ (see May [5, p. 12] for definition of homotopy). Q.E.D.

COROllary 8. If $\varphi$ and $\varphi^{\prime}: \Lambda \rightarrow \Gamma$ are homotopic, then so are the continuous maps $B \varphi$ and $B \varphi^{\prime}$.

COROLlary 9. If $\varphi$ and $\varphi^{\prime}: \Lambda \rightarrow \Gamma$ are homotopic, then $\varphi_{*}=\varphi_{*}^{\prime}: H_{*}(\Lambda) \rightarrow$ $H_{*}(\Gamma)$ where $H_{*}(\Lambda)$ is the homology group of $\Lambda$ with coefficients in $Z$ (i.e., $\operatorname{Tor}_{*}^{\wedge}(Z, Z)$ ).

Proof. The chain complex $C M \Lambda$ of $M \Lambda$ is exactly the chain complex induced by the bar resolution of $\Lambda$ with coefficient $Z$. (Cf. Lee [3] and [4].) Q.E.D.

Remark. Suppose $\Lambda$ is the singular category of a space $X$ and $\Lambda^{\prime}$ is the full subcategory whose objects consist of nondegenerate simplexes. Then $\Lambda$ and $\Lambda^{\prime}$ are homotopic equivalent in the sense that there are functors $\varphi: \Lambda \rightarrow \Lambda^{\prime}$ and $\varphi^{\prime} \Lambda^{\prime} \rightarrow \Lambda$ with $\varphi \circ \varphi^{\prime} \simeq 1_{\Lambda^{\prime}}$ and $\varphi \circ \varphi \simeq 1_{\Lambda}$.

THEOREM 10. Let $f$ and $g: X \rightarrow Y$ be continuous maps. If $f \simeq g$, then $\Lambda S(f) \simeq \Lambda S(g): \Lambda S(X) \rightarrow \Lambda S(Y)$.

We first prove

LEMMA 11. For each $n \geqq 0$, there is a continuous map $I_{n}: \Delta^{2 n+1} \rightarrow \Delta^{n} \times I$ such that the following diagram commutes for $k=0,1$.

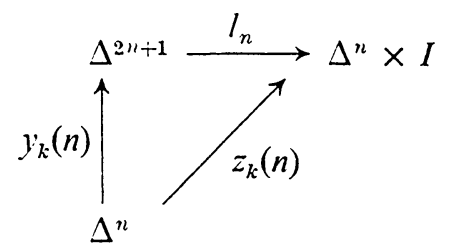


where $z_{k}(t)=(t, k)$ for $t \in \Delta^{n} ; y_{0}\left(t_{0}, \cdots, t_{n}\right)=\left(t_{0}, \cdots, t_{n}, 0, \cdots, 0\right) \in$ $\Delta^{2 n+1}$ and $y_{1}\left(s_{0}, \cdots, s_{n}\right)=\left(0, \cdots, 0, s_{0}, \cdots, s_{n}\right) \in \Delta^{2 n+1}$ for $\left(t_{0}, \cdots, t_{n}\right)$ and $\left(s_{0}, \cdots, s_{n}\right)$ in $\Delta^{n}$.

Furthermore, for each face map $x: \Delta^{m} \rightarrow \Delta^{n}$, there is a corresponding face map $\bar{x}: \Delta^{2 m+1} \rightarrow \Delta^{2 n+1}$ such that $l_{n} \circ \bar{x}=(x \times I) \circ l_{m}$ and $y_{k}(n) \circ x=\bar{x} \circ y_{k}(m)$ for $k=0,1$, i.e., the diagrams below are commutative

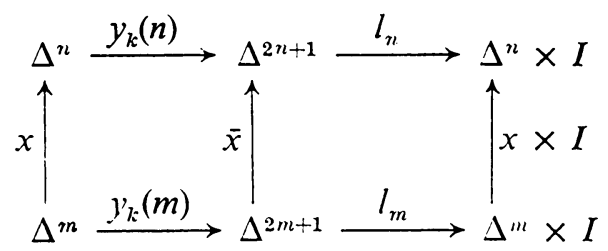

Proof. Define $l_{n}$ by

$$
l_{n}\left(t_{0}, \cdots, t_{n}, s_{0}, \cdots, s_{n}\right)=\left(\left(t_{0}+s_{0}, \cdots, t_{n}+s_{n}\right), 1-\sum t_{i}\right)
$$

for $\left(t_{0}, \cdots, t_{n}, s_{0}, \cdots, s_{n}\right)$ in $\Delta^{2 n+1}$. The image of $l_{n}$ is in $\Delta^{n} \times I$ because $\sum\left(t_{i}+s_{i}\right)=1$ and $0 \leqq 1-\sum t_{i} \leqq 1$. It is not hard to see that $l_{n}$ is the desired map.

Without loss of generality we may assume

\section{Define}

$$
x\left(t_{0}, \cdots, t_{m}\right)=\left(t_{0}, \cdots, t_{m}, 0, \cdots, 0\right) \in \Delta^{n} .
$$

$\bar{x}\left(t_{0}, \cdots, t_{m}, s_{0}, \cdots, s_{m}\right)=\left(t_{0}, \cdots, t_{m}, 0, \cdots, 0, s_{0}, \cdots, s_{1 m}, 0, \cdots, 0\right)$

for $\left(t_{0}, \cdots, t_{m}, s_{0}, \cdots, s_{m}\right) \in \Delta^{2 m+1}$ and it works. Q.E.D.

ProOF OF THEOREM 10. It suffices to show $\Lambda S\left(i_{0}\right) \simeq \Lambda S\left(i_{1}\right): \Lambda S(X) \rightarrow$ $\Lambda S(X \times I)$ where $i_{0}(t)=(t, 0)$ and $i_{1}(t)=(t, 1)$ for $t \in X$. We shall denote $\Lambda S\left(i_{k}\right)$ again by $i_{k}$ for $k=0,1$, and denote $\Lambda S(X)$ by $\Lambda, \Lambda S(X \times I)$ by $\Gamma$. For $u$ in $|\Lambda|$ with $\operatorname{dim} u=n$, we let $h(u)$ to be the $(2 n+1)$-singular simplex $(u \times I) \circ l_{n}$ in $|\Gamma|$.

$$
\Delta^{2 n+1} \stackrel{l_{n}}{\longrightarrow} \Delta^{n} \times I \stackrel{u \times I}{\longrightarrow} X \times I .
$$

For any morphism $x$ in $\Lambda(v, u)$, i.e., $x$ is a face map from $\Delta^{m}$ to $\Delta^{n}$ with $u \circ x=v$, we let $h(x): \Delta^{2 m+1} \rightarrow \Delta^{2 n+1}$ be the face map $\bar{x}$ of Lemma 11. Then we have $h(v)=(v \times I) \circ l_{m}=((u \circ x) \times I) \circ l_{m}=(u \times I) \circ(x \times I) \circ l_{m}=(u \times I) \circ$ $l_{n} \circ \bar{x}=h(u) \circ h(x)$, i.e., $h(x)$ is a morphism from $h(v)$ to $h(u)$. Thus we have defined, modulo some minor verifications, a covariant functor from $\Lambda$ to $\Gamma$.

To complete the proof, we need only exhibit two natural transformations $\eta_{k}: i_{k} \rightarrow h$ for $k=0,1$. For $u \in|\Lambda|$ with $\operatorname{dim} u=n$, we let $\eta_{k}(u)$ to be the 
face map $y_{k}(n): \Delta^{n} \rightarrow \Delta^{2 n+1}$ of Lemma 11. Since $i_{k}(u)=i_{k} \circ u=(u \times I) \circ z_{k}=$ $(u \times I) \circ I_{n} \circ y_{k}=h(u) \circ y_{k}$, it follows that $\eta_{k}(u)$ is a morphism from $i_{k}(u)$ to $h(u) . \eta_{k}$ is indeed a natural transformation because for any $x \in \Lambda(v, u)$ we have $\eta_{k}(u) \circ i_{k}(x)=y_{k}(n) \circ x, h(x) \circ \eta(v)=\bar{x} \circ y_{k}(m)$, where $n=\operatorname{dim} u$ and $m=\operatorname{dim} v, y_{k}(n) \circ x=\bar{x} \circ y_{k}(m)$ and hence $\eta(u) \circ i_{k}(x)=h(x) \circ \eta(v)$. Q.E.D.

Proof of THEOrem 1. (a) Theorem 10 shows that $f \simeq g$ implies $\Lambda S(f) \simeq \Lambda S(g)$. Conversely we suppose $\Lambda S(f) \simeq \Lambda S(g)$, then by Corollary 8 we have $T M \Lambda(f) \simeq T M \Lambda(g)$ as continuous maps. It follows from Proposition 5 that the following diagram is commutative.

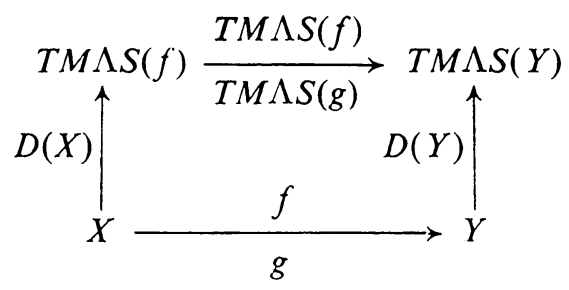

Since $X$ and $Y$ are $\mathrm{CW}$ complexes, $D(X)$ and $D(Y)$ are homotopy equivalences. Thus $f$ and $g$ are homotopic because $T M \Lambda S(f)$ and $T M \Lambda S(g)$ are. (b) is a direct consequence of (a). Q.E.D.

OPEN QUESTIONS. (1) For a given functor $\varphi: \Lambda S(X) \rightarrow \Lambda S(Y)$, is there a continuous map $f: X \rightarrow Y$ such that $\Lambda S(f) \simeq \varphi$ ? (2) Does the functor $\Lambda$ preserve homotopy? We expect to have a negative answer for (1), but (2) should be true at least for maps between Kan complexes.

\section{REFERENCES}

1. D. W. Anderson, Simplicial K-theory and generalized homology theories. I (preprint).

2. D. M. Kan, On c.s.s. complexes, Amer. J. Math. 79 (1957), 449-476. MR 19, 759.

3. M. J. Lee, A generalized Mayer-Vietoris sequence (submitted).

4. - Homology of small categories and its applications, Thesis, University of Rochester, 1971.

5. J. P. May, Simplicial objects in algebraic topology, Van Nostrand Math. Studies, no. 11, Van Nostrand, Princeton, N.J., 1967. MR 36 \#5942.

6. S. Okamoto, Partially ordered sets and semi-simplicial complexes, Math. Japon. 15 (1970), 69-79.

7. G. Segal, Classifying spaces and spectral sequences, Inst. Hautes Études Sci. Publ. Math. No. 34 (1968), 105-112. MR 38 \#718.

Department of Mathematics, louisiana State University, Baton Rouge, LOUISIANA 70803

Current address: Department of Mathematics, Louisiana State University in New Orleans, New Orleans, Louisiana 70122 